\title{
MODELLING ASYMMETRICAL SYNCHRONIZATION WITH DOMINANT AGENTS
}

\author{
K. SiekAŃski ${ }^{\mathrm{a}}$, P.F. Góra ${ }^{\mathrm{a}, \mathrm{b}}$ \\ aThe Marian Smoluchowski Institute of Physics, Jagiellonian University \\ Reymonta 4, 30-059 Kraków, Poland \\ ${ }^{b}$ Mark Kac Complex Systems Research Centre, Jagiellonian University \\ Reymonta 4, 30-059 Kraków, Poland
}

(Received July 22, 2014)

We present an extension of the classical Kuramoto model, adjusted for analysis of sets of asymmetrically coupled units. Results of numerical simulations suggest that violating Newton's Third Law by adding a strongly dominating unit can lead to synchronization in systems previously unable to synchronize.

DOI:10.5506/APhysPolB.45.1915

PACS numbers: 05.45.Xt

\section{Introduction}

Spontaneous synchronization of coupled oscillators is an enormously common phenomenon in virtually all areas of science [1, 2]. The most commonly used model of synchronization, proposed by Kuramoto, describes the mean field approximation [3-8]. However, this approximation does not cover all oscillatory systems that exist in nature. Therefore, several other cases have been investigated. One of the most widely discussed generalizations of the Kuramoto model applies to systems of oscillators connected by various types of network $[9,10]$. Many important aspects of synchronization phenomena have also been thoroghly discussed by Callenbach et al. [11].

This article concentrates on situations when interactions in a set of oscillators are not symmetrical: Newton's Third Law is violated. This represents systems in which information between units is transferred in one direction (e.g. neurons connected through synapses) or units are not equal (ecological systems, financial markets etc.). 


\section{Model}

\subsection{Modified Kuramoto equation}

We postulate a set of $N$ differential equations (based on a modified $\mathrm{Ku}$ ramoto model [3]) describing the coupling of all $N$ oscillators

$$
\frac{d \theta_{i}}{d t}=\omega_{i}+K \sum_{j=1}^{N} \frac{c_{j}}{c_{i}} \sin \left(\theta_{j}-\theta_{i}\right), \quad i=1,2,3, \ldots, N,
$$

where: $N$ - number of oscillators, $\theta_{i}$ - phase of oscillator $i, \omega_{i}$ - natural frequency of oscillator $i$ (Gaussian-distributed), $c_{i}$ - 'influence constant' of oscillator $i, K$ - coupling constant. Obviously, if all influence constants $c_{i}$ are equal, the equation reduces to its standard version.

\subsection{Influence constants}

A natural assumption is that influence constants should be described by Gaussian distribution $\sigma$. However, avoidance of singularities requires them to be positive. Therefore, we assume truncated normal distribution with positive mean and standard deviation not exceeding half of the mean. Thus, if a random $c_{k}$ constant happens to be zero or negative, it can be removed and the distribution does not cease to be approximately Gaussian.

\subsubsection{Order parameter}

To describe the state of the system, we will use the complex order parameter [1]

$$
r=\frac{1}{N} \sum_{j=1}^{N} e^{i \theta_{j}}
$$

Synchronization is full, when $|r|=1$, and the less $|r|$ is, the more desynchronised the system becomes. At $|r| \longrightarrow 0$, there is complete desynchronization. Obviously, $|r|^{2}=1$ iff $\forall i, j \theta_{i}=\theta_{j}$, which is exactly the state of full synchronization.

\section{Synchronization}

This article focuses on two subjects: influence of asymmetry of oscillators on their synchronization and the effect that a dominating unit gives on the rest of population. Domination of $k^{\text {th }}$ oscillator (in this context) is represented by its influence constant $c_{k} \gg c_{i} \forall i \neq k$. 


\subsection{Parameters and assumptions}

Due to the complexity of the discussed problem, the simplest way of examining it is numerical simulation based on idea of integrating equations (1) using trapezoidal method. Therefore, there are three sets of random numbers required for every single simulation:

- Gaussian distribution of influence constants $c_{i}$ (with mean 1024.0 and standard deviation $\sigma$ );

- Gaussian distribution $g(\omega)$ of natural frequencies $\omega_{i}$ (with fixed mean 0.0 and standard deviation 1.0);

- uniform distribution of initial values of phases $\theta_{i} \in[0,2 \pi]$.

In all simulations, standard deviation $\sigma$ was limited to interval $[0,512]$ so as to avoid frequent generation of non-positive values of $c_{i}$.

\subsection{Critical coupling constant}

As mentioned previously, at $\sigma=0$ all influence constants are equal and equation (1) reduces to the Kuramoto model in which synchronization appears only when $K$ exceeds its critical value given by expression

$$
K_{\mathrm{C}}^{\mathrm{Th}}=\frac{2}{\pi g(0)} \approx 1.60
$$

where $g(\omega)$ is the distribution of natural frequencies. The first step of research was to calculate experimental value of critical coupling, which led to the result

$$
K_{\mathrm{C}}=1.65(25)
$$

which proves correct when compared to theoretical value (4).

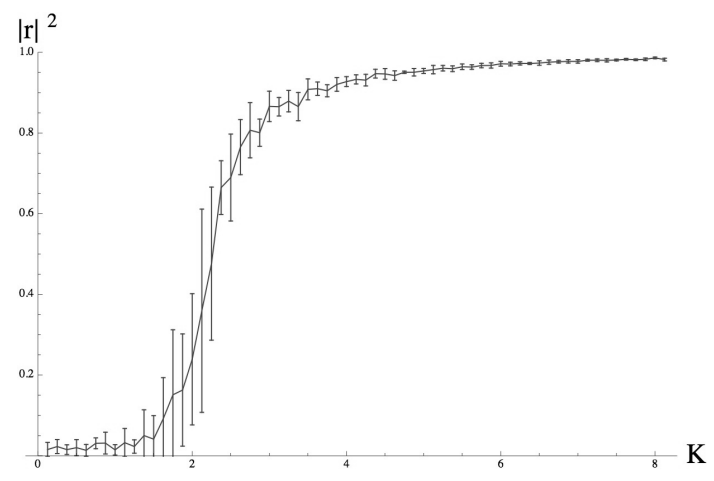

Fig. 1. Synchronization after long time for various values of coupling constant $K$. 
Figure 1 presents the character of critical coupling constant: after $K$ exceeds $K_{\mathrm{C}}$, the system quickly synchronizes, while for $K<K_{\mathrm{C}}$ synchronization drops nearly to zero.

\subsection{Influence of $\sigma$ on critical coupling constant}

Next, the Kuramoto model was disturbed by increasing $\sigma$. In order to investigate the influence of $\sigma$ on final state of the population, dependence of $|r|^{2}$ (synchronization) on $\sigma$ was calculated for various values of $K$, as shown in figure 2.

The main observation is that $\sigma$ chosen with respect to the above described assumptions does not significantly change final state of the system.

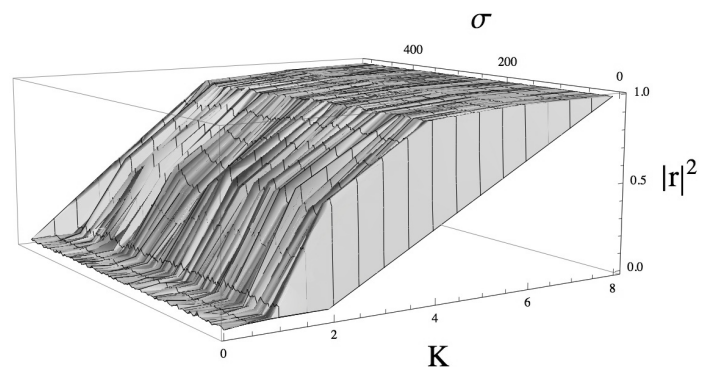

Fig. 2. Dependence of synchronization after long time on coupling constant $(K)$ and standard deviation of influence constants $(\sigma)$ in the case of no domination.

\section{Domination}

Another major question is what happens if one of the influence constants is a lot larger than the mean of distribution of other influence constants. For

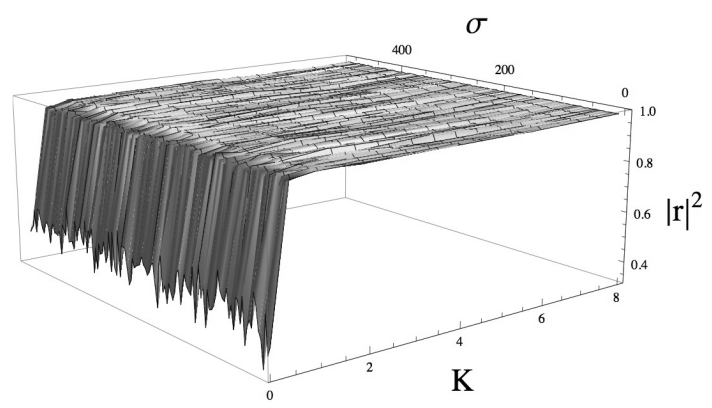

Fig. 3. Dependence of synchronization after long time on coupling constant $(K)$ and standard deviation of influence constants $(\sigma)$ in presence of a dominating unit. 
presented simulations, we assumed the dominating unit to be 1024 times larger than the mean. Figure 3 shows how synchronization depends on $\sigma$ and $K$ when the system is supplemented by a dominating unit.

It is visible that range of synchronization expands (compared to the case of no domination) - critical coupling $K_{\mathrm{C}}$ lowers. The main conclusion is that a dominating unit is capable of leading the population to synchronization, even if the latter is not possible in the case of classical mean field coupling.

\section{Conclusions}

Presented results indicate that, after sufficiently long time of interaction, a strongly dominating unit in a set of coupled oscillators leads to phase synchronization. This effect is irrespective of standard deviation of individual influence constants, as long as they are approximately Gaussian-distributed among positive numbers.

\section{REFERENCES}

[1] S.H. Strogatz, Physica D 143, 1 (2000).

[2] S.H. Strogatz, SYNC: the Emerging Science of Spontaneous Order, Hyperion, NY 2003.

[3] Y. Kuramoto, in: H. Arakai (Ed.), International Symposium on Mathematical Problems in Theoretical Physics, Lect. Notes Phys., Vol. 39, Springer, New York 1975, p. 420.

[4] Y. Kuramoto, Chemical Oscillations, Waves, and Turbulence, Springer, Berlin 1984.

[5] Y. Kuramoto, Progr. Theor. Phys. Suppl. 79, 223 (1984).

[6] H. Sakaguchi, Y. Kuramoto, Progr. Theor. Phys. 76, 576 (1986).

[7] Y. Kuramoto, I. Nikishawa, J. Stat. Phys. 49, 569 (1987).

[8] Y. Kuramoto, I. Nikishawa, in: H. Takayama (Ed.), Cooperative Dynamics in Complex Physical Systems, Springer, Berlin 1989.

[9] M. Chen, J. Kurths, Dynamical Optimization and Synchronization in Adaptive Complex Networks, in: Adaptive Networks Theory, Models and Applications, T. Gross, H. Sayama (Ed.), Springer, Dordrecht, New York 2009, p. 165.

[10] J. Ochab, P.F. Góra, Acta Phys. Pol. B Proc. Suppl. 3, 453 (2010) .

[11] V.S. Anishchenko et al., Nonlinear Dynamics of Chaotic and Stochastic Systems: Tutorial and Modern Developments, Springer, Heidelberg, Berlin 2007.

[12] W.H. Press, S.A. Teukolsky, W.T. Vetterling, B.P. Flannery, Numerical Recipes: The Art of Scientific Computing, Third Edition, New York: Cambridge University Press, 2007.

[13] L. Callenbach et al., Phys. Rev. E65, 051110 (2002). 